Research report

\title{
Reduced processing of alcohol cues predicts abstinence in recently detoxified alcoholic patients in a three-month follow up period: An ERP study
}

\author{
Géraldine Petit ${ }^{a}, *$, Agnieszka Cimochowska ${ }^{\mathrm{a}}$, Carlos Cevallos ${ }^{\mathrm{b}}$, Guy Cheron ${ }^{\mathrm{b}}$, \\ Charles Kornreich ${ }^{\mathrm{a}}$, Catherine Hanak ${ }^{\mathrm{a}}$, Elisa Schroder ${ }^{\mathrm{a}}$, \\ Paul Verbanck ${ }^{\mathrm{a}}$, Salvatore Campanella ${ }^{\mathrm{a}, * *}$ \\ a Laboratory of Psychological Medicine and Addictology, ULB Neuroscience Institute (UNI), Université Libre de Bruxelles (ULB), Brussels, Belgium \\ ${ }^{\mathrm{b}}$ Laboratory of Neurophysiology and Movement Biomechanics, ULB Neuroscience Institute (UNI), Université Libre de Bruxelles (ULB), Brussels, Belgium
}

\section{H I G H L I G H T S}

- Early alcohol abstainers show different electrophysiological features compared to relapsers.

- Abstainers show decreased P3 amplitude for alcohol compared to non-alcohol related pictures.

- The decreased P3 could express a reduction of the motivational significance of alcohol pictures.

- The difference in amplitude between alcohol and non-alcohol cues is the best predictor of relapse.

- Reaction times do not allow differentiating between abstainers and relapsers.

\section{A R T I C L E I N F O}

\section{Article history:}

Received 5 September 2014

Received in revised form

29 December 2014

Accepted 31 December 2014

Available online 8 January 2015

\section{Keywords:}

Alcohol dependence

Relapse

Attentional bias

ERPs

P3

SLORETA

\begin{abstract}
A B S T R A C T
One of the major challenges in alcohol dependence is relapse prevention, as rates of relapse following detoxification are high. Drug-related motivational processes may represent key mechanisms in alcoholic relapse. In the present study, event-related potentials (ERPs) were recorded during a visual oddball task administered to 29 controls ( 11 females) and 39 patients ( 9 females). Deviant stimuli were related or unrelated to alcohol. For patients, the task was administered following a 3-week detoxification course. Of these, 19 relapsed during the three months follow-up period. The P3, an ERP component associated with activation of arousal systems in the brain and motivational engagement, was examined with the aim to link the fluctuation of its amplitude in response to alcohol versus non-alcohol cues to the observed relapse rate. Results showed that compared to relapsers, abstainers presented with a decreased P3 amplitude for alcohol related compared to non-alcohol related pictures $(p=.009)$. Microstate analysis and sLORETA topography showed that activation for both types of deviant cues in abstainers originated from the inferior and medial temporal gyrus and the uncus, regions implicated in detection of target stimuli in oddball tasks and of biologically relevant stimuli. Through hierarchical regression, it was found that the P3 amplitude difference between alcohol and non-alcohol related cues was the best predictor of relapse vulnerability $(p=.013)$. Therefore, it seems that a devaluation of the motivational significance of stimuli related to alcohol, measurable through electrophysiology, could protect from a relapse within three months following detoxification in alcohol-dependent patients.
\end{abstract}

(C) 2015 Elsevier B.V. All rights reserved.

\footnotetext{
* Corresponding author at: CHU Brugmann, Laboratory of Psychological Medicine and Addictology, 4 Place Vangehuchten, 1020 Brussels, Belgium. Tel.: +32 24772851. ** Corresponding author at: The Belgian Fund for Scientific Research (FNRS), CHU Brugmann, Department of Psychiatry, 4 Place Vangehuchten, 1020 Brussels, Belgium. Tel.: +32 24773465 .

E-mail addresses: geraldine.petit@chu-brugmann.be (G. Petit), salvatore.campanella@chu-brugmann.be (S. Campanella).
}

\section{Introduction}

Although the first step in the treatment of alcohol dependence (detoxification) is straightforward, a major challenge is the prevention of relapse. Typically, around 50\% of patients drop out of treatment and resume alcohol use within three months of the end of detoxification [1]. From a clinical point of view, it is essential to identify factors influencing treatment outcome and relapse that 
may be modulated by therapeutic intervention [2]. A potential key element is that of attentional bias [3,4]. Theoretical accounts underlying attentional bias rely on the incentive sensitization theory of Robinson and Berridge [5]. The theory assumes that drugs of abuse can induce neuroadaptations in incentive motivation and reward systems, causing these systems to become hypersensitive to both drugs and drug-related stimuli. The incentive salience attributed to drug-related stimuli is responsible for the attention to be automatically oriented toward these stimuli: this is called an attentional bias. Many studies have indeed demonstrated the presence of a cognitive processing bias for alcohol-related stimuli in alcohol dependence. Historically, authors used behavioral assessment techniques through modified neuropsychological tasks (such as Stroop Task or Dot Probe Detection Task) in which they showed altered reaction times in response to alcohol-related stimuli [6-12] or difficulty shifting attention away from them [13-17] in alcohol abusers. Today, these behavioral techniques are used as training instruments dedicated to overcoming these biases. This relatively new area of investigation has yielded promising results (see [18] for a review) and is very relevant given that the phenomenon of cue reactivity is believed to play a central role in the maintenance of drug consumption disorders $[19,20]$ and relapse $[3,21-25]$. Indeed, attentional biases are believed to elicit conditioned responses such as drug craving and consumption (e.g. [26]). Moreover, Robinson and Berridge proposed that an important characteristic of the phenomenon of sensitization underlying attentional bias is that it survives long after taking the drug has stopped [5]. It has been shown in animal, as well as human studies, that sensitization could still be present years after the subject has stopped using drugs $[27,28]$. This characteristic of conditioned associations and attentional bias could explain the phenomenon of relapse after a long period of abstinence (e.g. [3,29]).

Many researchers have examined the association between bias toward alcohol-related stimuli and craving (e.g. [30]). However, very few have evaluated the critical clinical question of the extent to which the presence (or absence) of attentional bias can predict the likelihood of relapse (or remaining abstinent) in people trying to abstain. To our knowledge, only two studies based on behavioral investigations of attentional bias related a link between their presence and alcohol relapse [11,31]. Concerning dependencies on other substances, a link between bias and treatment outcomes has been found in some studies [32-34], but the predictive value could not be confirmed in the same amount of work [34-36]. New developments in cognitive neuroscience provide an opportunity to investigate additional aspects of relapse predictors as processing bias in a way that go beyond observable behavioral measures [37,38]. Neurosciences techniques, such as event-related potentials (ERPs) of the electroencephalogram (EEG), have shown their potential to highlight some brain abnormalities not detectable based on behavioral data only (e.g. [39]). These techniques are able to reveal the neural underpinnings of the cognitive configuration and processing response to stimuli linked to substance use $[40,41]$. The use of the ERP technique has objectified that the cerebral activity recorded during processing of alcohol-related pictures is heightened in alcoholics patients compared to controls. Specifically, researchers have shown that the P300 component (also called P3) in response to alcohol-related stimuli is heightened in alcohol abusers compared to controls [42,43]. The P3 component is a large, positive deflection of the ERP, arising about $300-800 \mathrm{~ms}$ after stimulus presentation, typically maximal at the medial central and parietal electrode sites (e.g. [44,45]). It is believed to reflect the mental processes underlying the deployment of attentional resources to task-relevant stimuli (e.g. $[44,46])$. Additionally, research has repeatedly shown that the amplitude of the P3 is enhanced in response to stimuli that are necessary for survival of the individual and that tend to automatically attract attention, i.e., stimuli that signal threat or danger or that signal the availability of sex and food or reward (for a review, see [47]). Increased P3 amplitude has thus been correlated with motivational engagement, motivated attention, and the activation of arousal systems in the brain $[48,49]$. Therefore, the P3 enhancement in alcohol abusers compared to controls could indicate high "motivational" value of alcohol-related stimuli and reflect the allocation of attentional resources to stimuli corresponding to alcohol-dependent subjects' motivational states. Such P3 amplitude enhancement in response to motivationally relevant stimuli are classical in substance use disorders (see [50] for a review), as increased P3 has (as compared to neutral stimuli) also been shown for instance for food targets in obese individuals [51] as well as for drug cues in opiate addiction [52]. However, no study to date has investigated whether an increased (or reduced) P3 found in substance-dependent subjects may be a predictor of relapse (or abstinence). The main goal of this study is to examine whether an electrophysiological index of alcohol cue reactivity, the modulation of the amplitude of the P3 component, is predictive of alcohol relapse or abstinence in alcoholdependent patients three months after the end of a detoxification course. Specifically, we hypothesized that a decreased reactivity to alcohol cues, indexed by a lower $\mathrm{P} 3$ in response to these cues measured at the end of the detoxification cure would reflect a decrease in motivation for alcohol and would therefore be associated with subsequent alcohol abstinence in the next three months followup period. The scalp topography of the P3 elicited by both alcohol and non-alcohol related cues in abstainers was further assessed by means of the brain electrical microstates technique and its cortical sources were evaluated by standardized low resolution brain electromagnetic tomography (sLORETA).

\section{Methods and materials}

\subsection{Participants}

Inpatients diagnosed with alcohol dependence according to Diagnostic and Statistical Manual of Mental Disorders (DSM-IV) [53] criteria were recruited as volunteers during the third week of their treatment from the Alcohol Detoxification Program of the Institute of Psychiatry, Brugmann Hospital (Brussels, Belgium). Healthy controls, gender- and age-matched to alcohol-dependent patients were also recruited. Healthy individuals whose alcohol consumption exceeded 14 standard drinks/week ( 7 for women) or 4 drinks/day ( 3 for women) were excluded because their consumption pattern placed them 'at risk' for alcohol-related problems according to the National Institute on Alcohol Abuse and Alcoholism [54]. Exclusion criteria for both groups included: current DSM-IV diagnosis of axis I disorders (other than alcohol dependence for patients); significant previous or current medical problems; visual impairment; head injury affecting the central nervous system; and current medical treatment that could interfere and/or reduce the cognitive ability to perform the task. In our study, patients ( $n=39,11$ females) were $20-68$ years-old, and controls ( $n=29$, 9 females) were $24-72$ years old. Control participants abstained from alcohol consumption for at least $24 \mathrm{~h}$ before testing. Participants in the patient group were tested prior to discharge, approximately 3 weeks after admission in detoxification. These three weeks of detoxification typically takes place as follows. Patients are accommodated in single rooms. Exits are prohibited during the first week and limited to the garden of the hospital. They are then limited to the surrounding park in the second week. Visits are allowed every day. The purpose of the detoxification course is physical and psychological alcohol withdrawal. Psychiatric, medical, family, and social assessments are obtained. Benzodiazepines (Diazepam ${ }^{\circledR}$ ) are given in regressive doses. An anti-craving 
medication is also proposed. The patient is supplemented with B vitamins and receives 1.51 of juice per day during the first week.

\subsection{Oddball task}

The task consisted of a visual oddball paradigm in which participants were confronted with one regularly repeated standard stimulus and deviant target ones. Deviant stimuli consisted of pictures of a man drinking different beverages. Three out of these beverages were alcohol-related (A) and three others were not (NA). Alcohol related beverages were beer, whiskey and wine, and non-alcohol ones were Sprite, Coca-Cola and Fanta. The frequent stimulus was the same man drinking water. These pictures were repeated in four blocks. In each block, 144 stimuli were presented: the frequent stimulus appeared 114 times (79\%), and the three deviant pictures for each condition (A and NA) each appeared five times (21\%), for a total of 15 deviant $A$ and 15 deviant NA stimuli in each block (i.e. and a total of 60 deviant of each category (A and NA) for the whole task). Each picture was presented for $800 \mathrm{~ms}$. A black screen was displayed between pictures for a random duration of 600-1000 ms (see Fig. 1). The participants had $1200 \mathrm{~ms}$ to answer from the onset of the stimulus. They were instructed to indicate as quickly as possible (but not at the cost of accuracy) the occurrence of any deviant stimulus with a right finger tap. The response times and percentages of correct answers were recorded.

\subsection{Procedure}

Informed written consent was first obtained from each participant. They received an explanation of the nature and duration of the study and were informed of what was expected from them. They were told that they were free to participate or not, as well as leave the study at any time without having to justify their decision and, for patients, without this influencing in any way the medical care they receive. Patients agreed to submit details and contact information of their regular doctor and a close relative in order to be contacted again in the months following hospitalization. The experiment then started. Participants were first instructed to rate their urge to drink alcohol at the commencement of the study using a 100-mm Visual Analog Scale [55]. Next, participants sat in a darkened room on a chair approximately $1 \mathrm{~m}$ from a computer screen. The oddball task was administered after a practice block. Following the task, participants were asked to assess their craving scale and complete the following questionnaires: the Alcohol Use Disorder Identification Test (AUDIT, Saunders, [56]; the State-Trait Anxiety Inventory (STAI [57]); the Beck Depression Inventory (BDI [58]); and a questionnaire about alcohol and other drug consumption (history, characteristics and degree of family history of alcoholism [FHA], if any). The local ethics committee at the Brugmann Hospital (“Comited'Ethique Hospitalier OM 026") approved our study.

\subsection{Relapse evaluation}

For alcohol-dependent individuals, a three-month follow-up was conducted to gather information about drinking behavior once the detoxification program had ceased. Relapse was defined as the reinstallation of a state of alcohol dependence according to DSM-IV criteria, and treated as a binary outcome variable [59,60]. According to Wiers et al. [59], unique re-consumption that did not exceed three days and after which the patient recovered control without further negative consequences, was not defined as proper relapse. Information was obtained by telephone, supplied directly by patients and confirmed by family members, or the patient's regular doctor.

\subsection{EEG recording and analysis}

During the testing phase, EEG activity was recorded with 32 electrodes mounted on a Quik-Cap and placed in standard (based on the 10-20 system) and intermediate positions [61]. Recordings were made with a linked mastoid physical reference but re-referenced offline using a common average, based on the principle that the integral surface of the potential on a surface that completely encompasses all the active sources should be zero [62]. The EEG was amplified with battery-operated ANT ${ }^{\circledR}$ amplifiers with a gain of 30,000 and a bandpass of $0.01-100 \mathrm{~Hz}$. The impedance of all electrodes was maintained below $5 \mathrm{k} \Omega$. EEG was recorded continuously at a sampling rate of $1024 \mathrm{~Hz}$ with ANT Eeprobe software. 98.19\% of the participants' responses were correct (i.e., a finger tap given for deviant stimuli). Only correct answers were considered for analysis of reaction times and EEG activity. The trials contaminated by eye movements or muscular artifacts were manually eliminated offline. Epochs starting $200 \mathrm{~ms}$ before the onset of the stimulus and lasting for $800 \mathrm{~ms}$ were created. The data were filtered with a $30 \mathrm{~Hz}$ lowpass filter. To compute averages of P3 to target stimuli for each subject, two parameters were coded for each stimulus: (i) the type of stimulus (A; NA); and (ii) the type of response (key press for deviant stimulus, no key press for frequent stimulus). A general time window was first determined globally for the identification of the component of interest (the P3) based on the literature (e.g. $[63,64])$. The measurement window was then tailored for each participant: for each subject, the P3 was investigated by gathering individual values of maximum peak amplitudes and peak latencies for each stimulus type in a 350-650 ms time range. These data were obtained from the following electrodes: Pz, P3, P4, POz, Cz, and Fz.

\subsection{ERP statistical analysis}

We analyzed ERP and behavioral data using analysis of variance (ANOVA) and covariance (ANCOVA). Our aims were to investigate whether the group belonging (Patients vs. Controls or Relapsers vs. Non-relapsers) affected behavioral and electrophysiological responses to both stimuli types. To investigate whether the group variable affected reaction times in response to both stimuli types, a Type (A vs. NA) vs. Group (Relapsers, Non-Relapsers, and Controls), ANOVA was performed on reaction times for correct answers. To investigate whether the group variable affects the P3 parameters in response to both stimuli types, $2 \times 3 \times 6$ ANOVAs were performed separately for P3 latencies and amplitudes, imputing type of stimulus (A vs. NA) and electrodes (Pz, P3, P4, CP1, CP2 and $\mathrm{POz}$ ) as within-subject variables and group (Relapsers, NonRelapsers, and Controls) as the between-subject variable. Simple effects were explored and interaction sources were systematically examined using Student's independent or paired $t$-tests. Moreover, analyses were systematically redone to control for a possible influence of demographic variables that differed between groups and that are known to either influence cue reactivity and/or P3 component parameters i.e., family history of alcoholism $[65,66]$, anti-craving medication (e.g. [67,68]), tobacco smoking (e.g. [69]) and education level. Greenhouse-Geisser correction was applied to all ANOVAs and ANCOVAs when necessary. Hierarchical multiple regression analysis was used to examine predictors of abstinence within the follow-up period. All analyses were conducted with SPSS $17.02 \mathrm{H}$, with the level of significance at 0.05 .

\subsection{Microstate analysis}

In order to explore brain sources of the ERP effects observed, scalp ERP data were analyzed with the microstate analysis method introduced by Lehman et al. [70]. Briefly, it consists of calculating the global field power (GFP) corresponding to the standard 


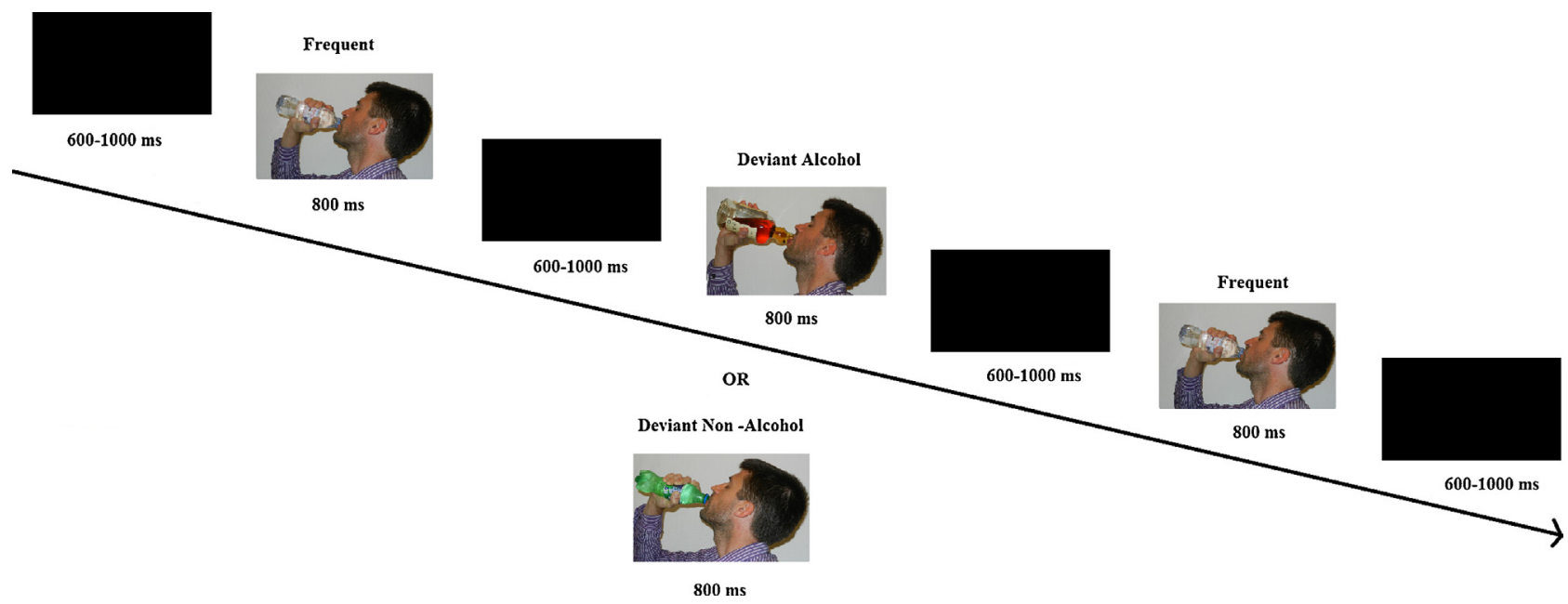

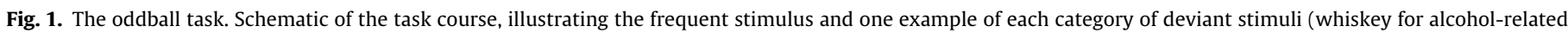
and Sprite for non-alcohol-related stimuli).

deviation of the averaged signals of all 32 electrodes recorded at each time point. For this we used publicly available CARTOOL software [71] dedicated for EEG microstate analysis on averaged pre-processed data. Then, a subroutine of CARTOOL (TAAHC) validated by Tibshirani and Walther [72] was used in order to perform a topographic atomize and agglomerate hierarchical clustering (TAAHC) to segment the data into microstates. This analysis was made on grand mean ERP across deviant cues (alcohol and nonalcohol) in the Non-relapsers' group.

\subsection{SLORETA analysis}

We performed source analysis on the defined microstates using sLORETA software. This method calculates a particular solution of the non-unique EEG inverse solution [73]. The sLORETA algorithm is based on the assumption that neighboring voxels tend to activate synchronously with each other [74]. This distributed inverse solution method is an interesting tool for modeling spatially distinct sources without prior knowledge about the anatomical location of the generators. In order to map the generators of P3 for deviant cues, we computed the sLORETA solution for the microstate containing the $\mathrm{P} 3$ activity. The sLORETA solution was computed using a 3D grid of points (or voxels) that represent possible sources of the signal, which are restricted to the gray matter based on the probabilistic brain tissue maps available from the Montreal Neurological Institute (MNI) [75]. Finally, the equally spaced grid points $(5.00-\mathrm{mm}$ grid spacing) and the recording array (32 electrodes) were placed in registration with the Collins 27 MRI produced by the MNI [76]. The final coordinates $(x, y, z$, Talairach coordinates) were used for labeling the corresponding brain areas [77].

\section{Results}

\subsection{Demographic and alcohol consumption characteristics}

Within the three-month follow-up period, 49\% (19/39) of patients relapsed. The clinical and demographic characteristics of Controls (C), Relapsers (R) and Non-relapsers (NR) are summarized in Table 1. As described in Table 1, no group differences were found for gender $\left(\chi^{2}=.128 ; p=.938\right)$ and age $(F(2,67)=2.967 ; p=.058)$.

Nevertheless, the groups differed concerning a few variables: education level $(F(2,67)=11.794 ; p<.001)$, for which both patients groups were lower than controls $(\mathrm{R}: t(33.596)=-4.748 ; p<.001$; NR: $t(31.401)=-3.327 ; p=.002)$; AUDIT score $(F(2,67)=198.377$; $p<.001)$, for which both patient groups scored higher than Controls as expected $(\mathrm{R}: t(25.498)=20.05 ; p<.001 ; \mathrm{NR}: t(22.614)=14.436$; $p<.001)$; craving score before experiment $(F(2,67)=5.101$; $p=.009$ ) for which Relapsers showed higher craving than Controls $(t(19.091)=2.279 ; p=.034)$ whereas Non-relapsers did not differ from Controls; craving score after experiment $(F(2,67)=3.681$; $p=.031$ ), for which Relapsers showed higher craving than Controls $(t(18.99)=2.191 ; p=.041)$ whereas Non-relapsers did not differ from Controls; global craving score $(F(2,67)=5.256$; $p=.008)$, for which Relapsers showed higher craving than Controls $(t(19.07)=2.385 ; p=.028)$ whereas Non-relapsers did not differ from Controls; alcohol intake prior detoxification $(F(2,67)=30.249$; $p<.001$ ), for which both patient groups were higher than Controls as expected $(\mathrm{R}: t(18.033)=6.991 ; p<.001 ; \mathrm{NR}: t(19.025)=5.922$; $p<.001)$; tobacco smoking, for which both patients groups had a greater number of smokers compared to Controls (R: $\left.\chi^{2}=26.716 ; p<.001 ; \mathrm{NR}: \chi^{2}=24.507 ; p<.001\right)$; depression score $(F(2,67)=15.144 ; p<.001)$, for which both patients scored higher than Controls (R: $t(40.126)=5.12 ; p<.001 ; N R: t(35.369)=4.2$; $p<.001)$; trait anxiety $(F(2,67)=6.598 ; p=.002)$, for which both patient groups scored higher than Controls (R: $t(44.747)=3.118$; $p=.003 ; \quad \mathrm{NR}: \quad t(39.310)=2.998 ; \quad p=.005) ; \quad$ state anxiety $(F(2,67)=9.148 ; p<.001)$, for which both patients scored higher than Controls (R: $t(40.941)=3.396 ; p=.002$; NR: $t(41.435)=3.738$; $p=.001$ ); and family history of alcoholism (FHA) for which both patients groups had a greater number of individuals with FHA compared to Controls (R: $\chi^{2}=4.907 ; p=.027$;R: $\chi^{2}=9.462 ; p=.002$ ).

As far as both patients groups (Relapsers and Non-relapsers) are concerned, no significant difference was found for age of onset of alcohol misuse $(t(36.834)=1.302 ; p=.201)$, duration of dependence $(t(36.619)=1.243 ; p=.222)$, number of prior detoxifications $(t(30.899)=1.504 ; p=.143)$, and benzodiazepine use $(t(34.823)=.111 ; p=.912)$. However, the groups differed for anticraving medication use $\left(\chi^{2}=5.132 ; p=.023\right)$ : the Relapsers group had a greater number of patients taking anti-craving medication compared to the Non-relapsers.

\subsection{Reaction times}

The analysis showed a significant effect for the type of stimulus $\left[F(1,65)=43.528, p<.001, \eta^{2}=.401\right.$, observed Power $\left.=1.000\right]$, suggesting that all participants had faster reaction times in response to A compared to NA stimuli $(493 \pm 80 \mathrm{~ms}$ vs. $511 \mathrm{~ms} \pm 82$ ) (see Table 2). A Type vs. group interaction was also found $\left[F(2,65)=5.299, p=.007, \eta^{2}=.140\right.$, observed Power $\left.=.820\right]$. 
1)

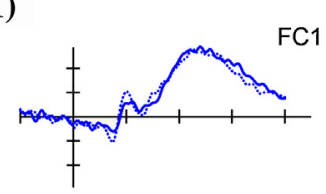<smiles>[3H]C([3H])([3H])N=NCCC=CC=CC</smiles>

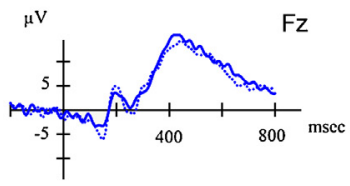<smiles>[Y]C([3H])([3H])C=CC=CC=C</smiles>

No amplitude difference

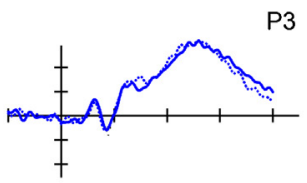

P3

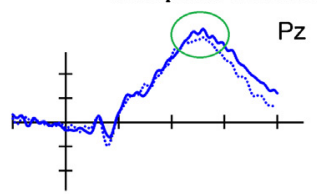

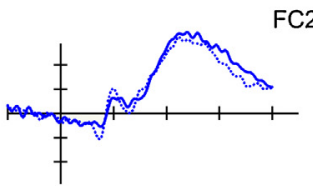

FC2
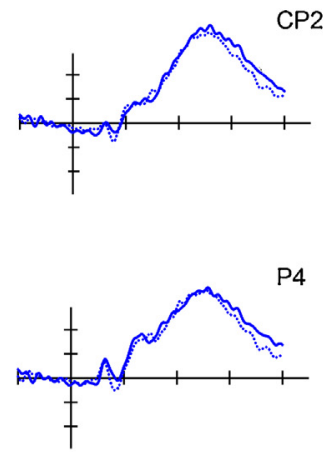

$\mathrm{POz}$

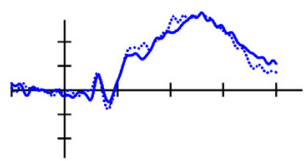

Relapsers - Non Alcohol Related cues

Non relapsers - Non AIcohol Related cues

Relapsers - Alcohol cues

Non relapsers - Alcohol cues

2)
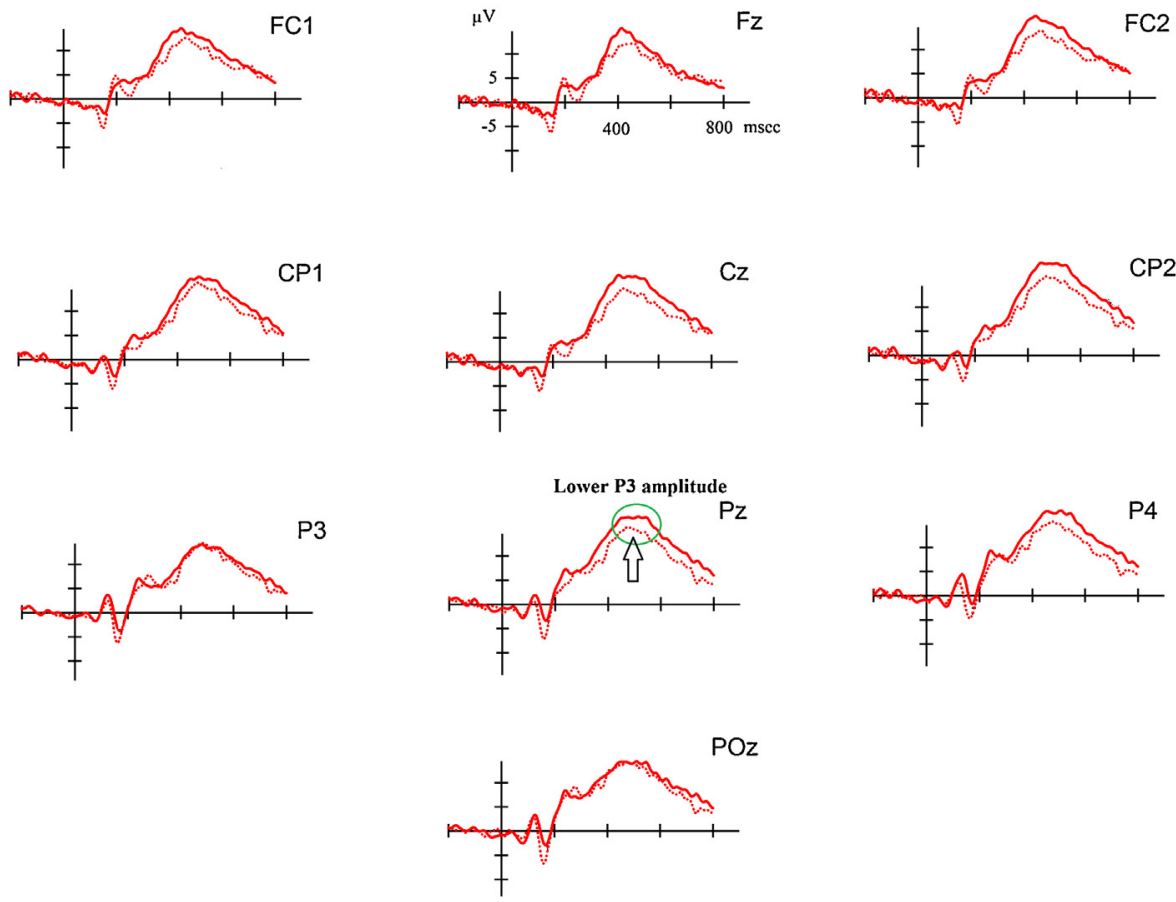

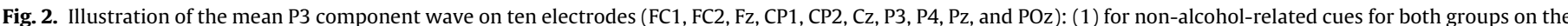

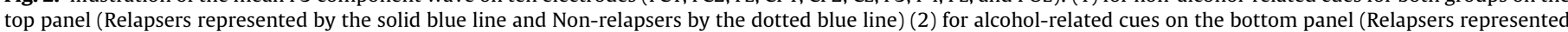

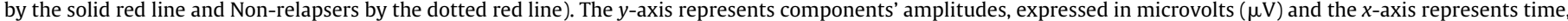
expressed in milliseconds (ms). 
Table 1

Clinical characteristics of Relapsers, Non-relapsers and Controls in our study presented as means (standard deviations).

\begin{tabular}{|c|c|c|c|}
\hline & Relapsers $(n=19)$ & Non-relapsers $(n=20)$ & Controls $(n=29)$ \\
\hline Females & 5 & 6 & 9 \\
\hline Age (years) & $43(11)$ & $51(12)$ & $44(12)$ \\
\hline Education level $^{\mathrm{a}}$ & $12(3)$ & $13(3)$ & $16(2)$ \\
\hline Alcohol problems (AUDIT) & $31(4)$ & $32(9)$ & $4(2)$ \\
\hline Alcohol intake prior detoxification (U/day) & $21(12)$ & $21(16)$ & $0.5(0.4)$ \\
\hline Age of onset of alcohol misuse $\mathrm{e}^{\mathrm{b}}$ & $31(10)$ & $35(12)$ & $\mathrm{NA}$ \\
\hline Duration of current treatment (days) & $18.7(2.4)$ & $19.2(1.7)$ & NA \\
\hline Duration of dependence ${ }^{c}$ (years) & $12(10)$ & $16(9)$ & NA \\
\hline Number of prior detoxifications & $1.4(3.0)$ & $3.4(5.1)$ & NA \\
\hline Smoking (\% with nicotine dependence) & 89 & 85 & 14 \\
\hline Depression (BDI score) & $22(9)$ & $21(12)$ & $7(9)$ \\
\hline Anxiety Trait (STAI-B) & $47(7)$ & $51(9)$ & $38(9)$ \\
\hline Anxiety State (STAI-A) & $47(8)$ & $48(9)$ & $40(9)$ \\
\hline Craving before experiment & $18(31)$ & $5(10)$ & $1(6)$ \\
\hline Craving after experiment & $17(31)$ & $7(18)$ & $1(6)$ \\
\hline Global craving score & $18(30)$ & $6(11)$ & $1(6)$ \\
\hline Family history of alcoholism ${ }^{\mathrm{d}}$ (\% with FHA) & 42 & 55 & 13 \\
\hline Anticraving medication (\% receiving treatment) & 84 & 50 & NA \\
\hline Benzodiazepine (\% of users) & 31 & 33 & NA \\
\hline
\end{tabular}

a Number of years of education since completing primary school.

b Age at which drinking first began to have an effect on the subjects' life of which he/she did not approve [99].

c Number of years that the subject fulfilled dependence criteria before assessment.

d Presence of at least one first- (father and/or mother) and/or second-degree (grandfather and/or grandmother) relative with alcohol dependence. AUDIT, Alcohol Use Disorders Identification Test.

One unit (U) represents $10 \mathrm{~g}$ of alcohol.

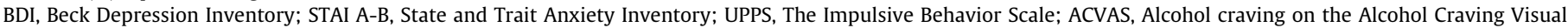
Analog Scales.

Independent $t$-tests showed that all groups differed in their reaction times between A and NA stimuli: reactions times to A stimuli were shorter than that of NA stimuli (Rs: $t(18)=-2.993 ; p=.008$; NRs: $t(19)=-5.577 ; p<.001 ; C: t(28)=-2.216 ; p=.035)$. Thus, to clarify the interaction, we performed a univariate ANOVA on the score of difference between reaction times to A vs. NA stimuli. It showed that while this score of difference did not differ between Controls and Relapsers (7.8 vs. 18.6; $p=.345$ ) and Relapsers and Non-relapsers (18.6 vs. $29.4 ; p=.441$ ), it significantly differed between Non-relapsers and Controls (29.4 vs. 7.8 ; $p=.006$ ). Controls had a smaller difference in their reaction times between $A$ and NA stimuli compared to Non-relapsers. However, when we secondarily controlled for the influence of the variables cited above using a Type (A vs. NA) vs. Group (Relapsers, Non-Relapsers, Controls) vs. medication (taking medication vs. not taking medication) vs. tobacco smoking (smoking vs. non-smoking) ANCOVA with education level as covariate, this effect was no longer significant $(p=.475)$.

\section{3. $P 3$}

\subsubsection{Amplitudes}

The analysis of P3 amplitudes did not show any main group effects, but it produced a significant group vs. type interaction $\left[F(1,65)=4.421, p=.016, \eta^{2}=.120\right.$, observed Power $\left.=.742\right]$. Paired $t$-test suggested that while in the Controls and the Relapsers' groups, no significant difference in response to the two types of stimuli was noted [R: $t(18)=1.184: 11.3(3.7)$ vs. $10.5(4.1) ; p=.252$;
C: $t(19)=-2.908 ; p=.143: 11.7$ (5.1) vs. $12.2(4.8)]$, in the Nonrelapsers group, A cues tended to elicit lower amplitudes than NA cues $[t(28)=-1.366 ; p=.009$ : 9.6 (5.7) vs. 11.3 (5.1)] (see Table 2 and Fig. 2). The additional analysis using a type (A vs. NA) vs. group (Relapsers, Non-relapsers, and Controls) vs. medication (taking medication vs. not taking medication) vs. tobacco smoking (smoking vs. non-smoking) ANCOVA with education level as covariate did not change these results $(p=.023)$.

\subsubsection{Latencies}

P3 latencies analyses did not show any group or interaction effect $(p>.085)$.

\subsection{Regression}

In order to examine the unique contribution of the difference between P3 amplitude for A stimuli and P3 amplitude to NA stimuli on abstinence beyond and above other variables, hierarchical regression was conducted with FHA, the craving, depression, and anxiety state scores, and the use of anti-craving medication entered as predictors in the first step. Together these variables could not significantly predict abstinence $(F(1,33)=1.554, p=.2)$. In a second step, we entered the difference between $\mathrm{P} 3$ amplitude for A stimuli and P3 amplitude to NA stimuli. The addition of this variable significantly contributed to the prediction of abstinence $(F(1,32)=6.869$, $p=.013)$. Thirty-three percent of the variance of the abstinence outcome was explained by the model and by this variable

Table 2

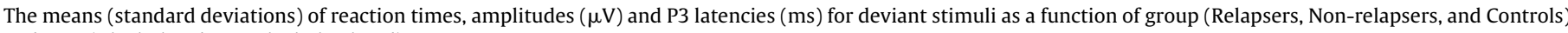
and type (Alcohol and Non-alcohol-related).

\begin{tabular}{|c|c|c|c|c|}
\hline & & Relapsers & Non-relapsers & Controls \\
\hline \multirow{2}{*}{ Reaction Times } & Alcohol-related pictures & $488(92)$ & $502(86)$ & $491(71)$ \\
\hline & Non-alcohol related pictures & $506(86)$ & $531(91)$ & $499(72)$ \\
\hline \multirow[t]{2}{*}{ P3 Amplitudes } & Alcohol-related pictures & $11.3(3.7)$ & $9.6(5.7)$ & $11.7(5.1)$ \\
\hline & Non-alcohol related pictures & $10.5(4.1)$ & $11.3(5.1)$ & $12.2(4.8)$ \\
\hline \multirow[t]{2}{*}{ P3 Latencies } & Alcohol-related pictures & $428(33)$ & $403(68)$ & $428(33)$ \\
\hline & Non-alcohol related pictures & $418(68)$ & $411(66)$ & $430(41)$ \\
\hline
\end{tabular}




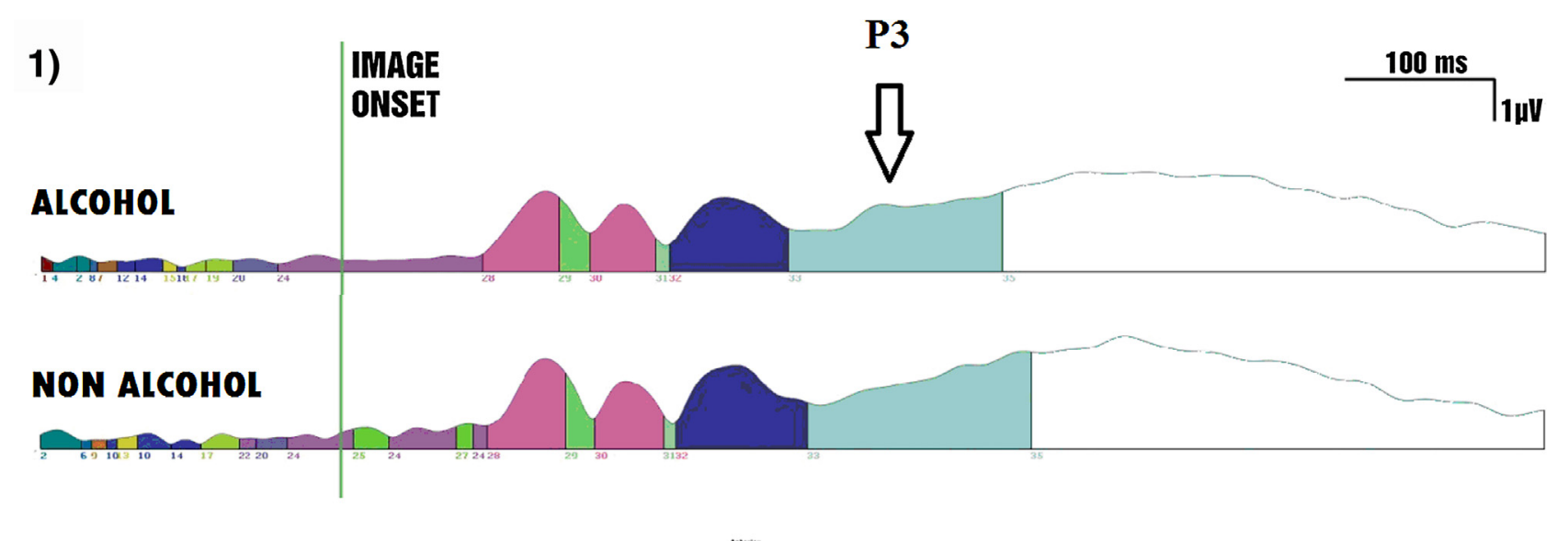

2)

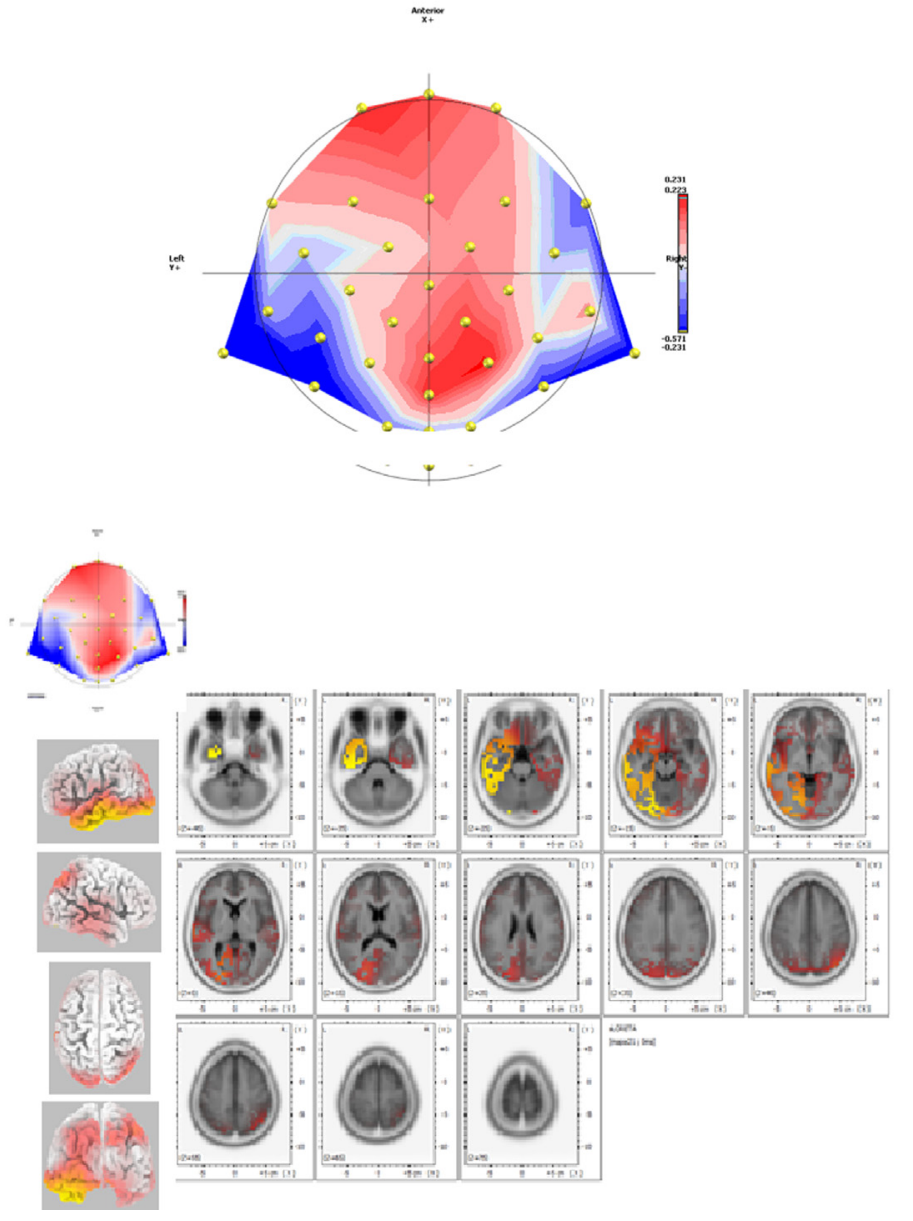

Fig. 3. Microstates and sLORETA analysis for alcohol- and non-alcohol-related stimuli in the Non-relapser group.

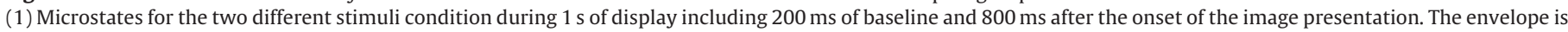

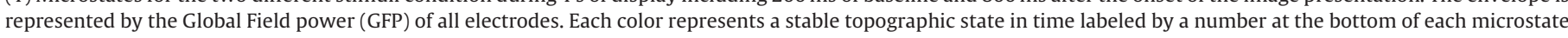

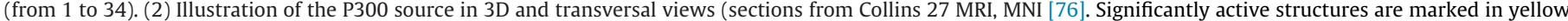

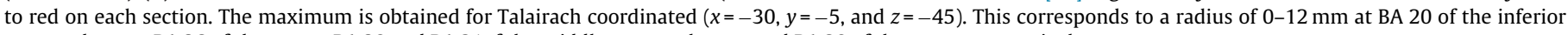
temporal gyrus, BA 36 of the uncus, BA 38 and BA 21 of the middle temporal gyrus and BA 28 of the uncus, respectively.

on its own. Odds of abstinence increased by a factor of 0.412 for each additional microvolt included in the difference between P3 amplitude for A stimuli and P3 amplitude to NA stimuli. Table 3 depicts the logistic regression results.

\subsection{Microstate analysis and sLORETA topography}

CARTOOL analysis performed on the averaged data demonstrated a series of microstates $(n=34)$, the duration of these microstates ranging from 5 to $300 \mathrm{~ms}$ (Fig. 3(1)). After the presentation of the deviant pictures, the microstates timing organization corresponding to cortical activation (since the microstate 28 relates to the $\mathrm{P} 1$ component) was similar for alcohol and nonalcohol related cues. We then focused our analysis on the time of interest, i.e., around the P3 (peaking at $403 \mathrm{~ms}$ and $411 \mathrm{~ms}$ for alcohol and non-alcohol cues, respectively) which was represented by the same microstate 33 . The localizations of the microstate 33 generators were found to be in the inferior and medial temporal gyrus (BA 20, BA 21, and BA 38) and the uncus (BA 28 and BA 36) (Fig. 3(2)). 
Table 3

Multiple hierarchical regression analysis examining predictors of abstinence after the three-month follow-up period.

\begin{tabular}{|c|c|c|c|}
\hline Variable & $\beta$ & S.E. & OR $(95 \% \mathrm{CI})$ \\
\hline \multicolumn{4}{|l|}{ Step 1} \\
\hline FHA & 0.166 & 0.158 & $0.166(-0.155$ to 0.488$)$ \\
\hline Medication & -0.341 & 0.172 & $-0.321(-0.690$ to 0.009$)$ \\
\hline Craving & -0.004 & 0.004 & $-0.210(-0.012$ to 0.003$)$ \\
\hline Depression & 0.003 & 0.008 & $0.068(-0.013$ to 0.019$)$ \\
\hline Anxiety & 0.001 & 0.010 & $0.017(-0.019$ to 0.021$)$ \\
\hline \multicolumn{4}{|l|}{ Step 2} \\
\hline FHA & 0.220 & 0.147 & $0.220(-0.080$ to 0.519$)$ \\
\hline Medication & -0.304 & 0.159 & $-0.287(-0.628$ to 0.019$)$ \\
\hline Craving & -0.002 & 0.004 & $-0.105(-0.009$ to 0.005$)$ \\
\hline Depression & -0.003 & 0.008 & $-0.680(-0.019$ to 0.012$)$ \\
\hline Anxiety & 0.002 & 0.009 & $0.035(-0.16$ to 0.20$)$ \\
\hline $\begin{array}{l}\text { Amplitude } \\
\text { NA-Amplitude A }\end{array}$ & 0.067 & 0.260 & $0.412(0.15-.119)$ \\
\hline
\end{tabular}

$\beta$, slope of the regression line; S.E., standard error; OR, odds ratio; $\mathrm{CI}$, confidence interval.

\section{Discussion}

The present results confirm that patients who remained abstinent during a three-month follow-up period can be differentiated from those who relapsed by a decrease of the P3 component amplitude in response to alcohol-related relative to neutral images. Most interestingly, this wave of difference between alcohol and nonalcohol pictures predicts abstinence during the follow-up period, above and beyond other relevant predictors such as family history of alcoholism, alcohol craving, depression and anxiety state scores, and the use of anti-craving medication. That is to say, a decrease in motivation for alcohol-related stimuli, which becomes not equal to that of neutral stimuli but still below the threshold thereof, could predict success in stopping alcohol consumption after detoxification.

Importantly, electrophysiological difference between relapsers and abstainers is observed while behavioral data (reaction times) do not allow differentiating between the groups. It thus seems that the use of electrophysiology allows circumventing the difficulty of behavioral measures to identify a link between relapse and attentional bias/cue reactivity phenomena. To our knowledge, no study to date has addressed the relationship between indices of electrophysiological response to stimuli associated with an abused substance and relapse in addictions. However, recent data from studies on the electrophysiological response to substance-related pictures in ex-addicted are interesting in light of our results. In the study of Littel et al. [78] alcohol-dependent patients in the sample were not just out of treatment at the time of the evaluation; however, they had an average of 5.3 months of abstinence, which makes them quite close to our group of Non-relapsers. And yet, data from Littel and colleagues suggest, identically to ours, that pictures associated with alcohol generate a decrease in the amplitude of the P3 component in these abstinent patients compared to controls. Two other studies have shown that exsmokers, exhibited significantly lower P3 and LPP (late positive potential) wave amplitudes in response to images related to cigarette smoking compared to those of current smokers [79,80], which also suggest that former (abstinent) smokers do not find cigarette-related cues to be as motivationally salient as present smokers.

Taken together, these data first seem to show that processing biases may not be a permanent feature of addiction. This is in contrast with a characteristic of the incentive-sensitization theory of Robinson and Berridge [5], which predicts that the neuro-adaptations underlying the bias for addiction cues are more or less permanent, and that addiction cues keep their incentive-motivational properties even after drug cessation. The reality appears to be more optimistic and the reverse plasticity phenomenon seems to be possible in some individuals. The incentive sensitization theory [5] suggests that addiction cue reactivity is due to the disturbance of a sensitized neural system (mesocorticolimbic dopamine and related systems) that normally works to attribute incentive salience to reward cues trough dopamine release. Via this dopamine liberation, the system converts cues associated with rewards into incentive stimuli, which renders them attractive and able to provoke an irrepressible need to obtain their reward. Repeated drug use produces a massive release of dopamine, inducing the sensitization of this brain system, leading to an increased drug wanting, in turn inducing excessive control of behavior by drug-related incentive stimuli. We could make the hypothesis that the decreased salience of addictionrelated stimuli in ex-addicted found in the studies cited above [78-80], and in alcohol-abstainers patients in the present study could indicate a normalization or a decrease of the attribution of incentive salience through the resumption of normal or diminished dopamine release in response to addiction cues. The occurrence of this phenomenon in some patients would protect them from a return to the addicted state. This remains, of course, hypothetical and should be properly tested by neurochemical studies.

In the meantime, our results secondly suggest that brain activity that processes alcohol-related stimuli might serve as a biomarker helping to identify patients vulnerable to relapse after detoxification, i.e., patients in whom this decreased salience of alcohol-related stimuli does not occur. The use of EEG as a screening instrument for relapse has gained much interest in recent years (e.g. [81-85]). Aside from new evidence indicating an advantage of its sensitivity compared to behavioral measures, the EEG is a relatively low-cost, non-invasive, and easily accessible biomarker. Therefore, its use in clinical settings to predict treatment issues in terms of alcohol consumption would be very profitable and achievable. However, it will require some time before we can use the marker identified in this study in clinical practice. Our results will have to be replicated on a larger scale to confirm that the factor we identified is associated with maintaining abstinence. Obviously, one of the biggest challenges will be the ability to translate associations between group characteristics and abstinence to individual predictions following treatment. If the results hold after replication and it is demonstrated that our tool can accurately forecast at the individual level, they could help identify dependent patients who are or are not prone to relapse. The systematic screening of the protective marker and its absence in some patients could then lead to offering these patients some cognitive approaches (e.g. [86,87]) and drug treatment (e.g. $[67,88,89]$ ) aimed to decrease responsiveness to stimuli associated with alcohol in addition to the usual psychological therapy. The microstate analysis allowed for the identification of stable topographic states during the presentation of deviant stimuli in the Non-relapsers' group. The timing was similar for both alcohol and non-alcohol related cues, which indicates highly conserved topography activation. The localization of the P3 generators of the microstate 33 was found to be in the inferior and medial temporal gyrus (BA 20, BA 21, and BA 38) as well as in the uncus (BA 28 and $B A 36)$. These regions have been associated with visual recognition and high-level visual processing (BA 20), emotional treatment (BA 21) [90], judgment process of visual stimuli (BA 28) [91] and face recognition (BA 36) [92]. Interestingly, temporal gyrus and uncus have been found to be activated by target stimuli in oddball tasks (e.g. $[93,94])$, by biologically relevant stimuli $[95]$ and by addictionrelated cues in alcohol-dependent populations [96]. The absence of differences in scalp topography and cortical sources between both alcohol and non-alcohol deviant cues suggests that the two types of stimuli activate the same neural processes in abstainers and only 
differ by the power of their activation, as indexed by the decreased amplitude of the P3 for alcohol-related cues.

The present study suffers from several limitations that should be taken into account in further studies. First, the dichotomous characteristic of the relapse variable did not allow us to more precisely investigate the frequency and quantity of relapsers' alcohol reconsumption during follow-up. Predictors of relapse as dichotomous measures could vary from predictors of the frequency and quantity of alcohol reconsumption. We also limited our investigation of reactivity toward information related to the visual aspect of alcohol. However, contact with information related to addictive substances is a fully sensitory experience, which may also include auditory, olfactory, tactile, etc. components. Some drinkers may be more sensitive to certain types of stimulatory channels than others and concurrent presentation of different types of stimulatory channels could also potentiate the observable effects. It seems that multi-sensory stimuli induce more robust brain responses than visual stimuli commonly used, and correlations between neuronal reactivity and clinical variables such as craving have been reported more frequently when using multi-sensory visual clues alone [97]. It is therefore possible that the lack of varietal stimuli channels used in our study could have led to an underestimation of the effects of alcohol-related stimuli. Finally, while the ERP analysis is a highly valuable, time-resolved, brain-imaging technique given its millisecond time-resolution and direct relationship to neuronal activity, it does not allow for making statements on localization. Although we used sLORETA to estimate the sources of scalp-recorded electric activity, the technique still presents some limitations (see [73] for a review), and especially in this work since we only based our analysis on 32 channels. Future studies utilizing simultaneous fMRI/EEG techniques would be able to provide both high spatial and time resolution and therefore more insight into specific neural generators of alcohol-cue elicited electrophysiological responses (e.g. [98]).

\section{Conclusion}

In conclusion, this study highlighted a neurophysiological protective marker of relapse, which consists of a reduction in the amplitude of the P3 component originating from the temporal gyrus and the uncus in response to the alcohol-related relative to non-alcohol related pictures, reflecting a decrease in motivation for alcohol related stimuli. In the future, it would therefore be advantageous to routinely assess the P3 amplitude in response to alcohol-related pictures compared to non-alcohol-related pictures in alcohol-dependent patients at the end of detoxification treatment. Ultimately, treatment programs could be tailored to individual patients to maximize their chances of success in abstinence.

\section{Role of the funding source and conflicts of interest}

The authors have no competing financial interest, potential conflict of interest, or financial relationship with commercial entities to report. The authors are funded by the Belgian Fund for Scientific Research (F.N.R.S., Belgium), but this funder did not exert any editorial direction or censorship on any part of this article.

\section{Acknowledgements}

Géraldine Petit and Salvatore Campanella are Research Fellow and Research Associate at the Belgian Fund of Scientific Research (FRS-FNRS), respectively.

\section{References}

[1] Finney JW, Hahn AC, Moos RH. The effectiveness of inpatient and outpatient treatment for alcohol abuse: the need to focus on mediators and moderators of setting effects. Addiction 1996;91:1773-96.

[2] Garland EL, Franken IHA, Howard MO. Cue-elicited heart rate variability and attentional bias predict alcohol relapse following treatment. Psychopharmacology 2012;222:17-26.

[3] Field M, Cox WM. Attentional bias in addictive behaviors: a review of its development, causes, and consequences. Drug Alcohol Depend 2008;97:1-20.

[4] Stritzke WG, Breiner MJ, Curtin JJ, Lang AR. Assessment of substance cue reactivity: advances in reliability, specificity, and validity. Psychol Addict Behav 2004:18:148-59.

[5] Robinson TE, Berridge KC, Addiction. Ann NY Acad Sci 2003;54:25-53.

[6] Johnsen BH, Laberg JC, Cox WM, Vaksdal A, Hugdahl K. Alcoholic subjects' attentional bias in the processing of alcohol-related words. Psychol Addict Behav 1994;8:111-5.

[7] Stetter F, Chaluppa C, Ackermann K. Alcoholics' selective processing of alcohol related words and cognitive performance on a Stroop task. Eur Psychiatry 1994:9:71-6.

[8] Bauer D, Cox WM. Alcohol-related words are distracting to both alcohol abusers andnon-abusers in the Stroop colour-naming task. Addiction 1998;93:1539-42.

[9] Stormark KM, Laberg JC, Nordby H, Hugdahl K. Alcoholics' selective attention to alcohol stimuli: automated processing? J Stud Alcohol Drugs 2000;61:18.

[10] Sharma D, Albery IP, Cook C. Selective attentional bias to alcohol related stimuli in problem drinkers and non-problem drinkers. Addiction 2001;96(2): 285-95.

[11] Cox WM, Hogan LM, Kristian MR, Race JH. Alcohol attentional bias as a predictor of alcohol abusers' treatment outcome. Drug Alcohol Depend 2002;68:237-43.

[12] Ryan F. Attentional bias and alcohol dependence: a controlled study using the modified Stroop paradigm. Addict Behav 2002;27:471-82.

[13] Stormark KM, Field NP, Hugdahl K, Horowitz M. Selective processing of visua alcohol cues in abstinent alcoholics: an approach-avoidance conflict? Addict Behav 1997;22(4):509-19.

[14] Noël X, Colmant M, Van Der Linden M, Bechara A, Bullens Q, Hanak C, et al. Time course of attention for alcohol cues in abstinent alcoholic patients: the role of initial orienting. Alcohol Clin Exp Res 2006;30:1871-7.

[15] Jones BT, Bruce G, Livingstone S, Reed E. Alcohol-related attentional bias in problem drinkers with the flicker change blindness paradigm. Psychol Addict Behav 2006;20:171-7.

[16] Townshend JM, Duka T. Avoidance of alcohol-related stimuli in alcoholdependent inpatients. Alcohol Clin Exp Res 2007;31:1349-57.

[17] Vollstädt-Klein S, Loeber S, von der Goltz C, Mann K, Kiefer F. Avoidance of alcohol-related stimuli increases during the early stage of abstinence in alcohol-dependent patients. Alcohol Alcohol 2009;44:458-63.

[18] Cox WM, Fadardi JS, Intriligator JM, Klinger E. Attentional bias modification for addictive behaviors: clinical implications. CNS Spectr 2014;19:215-24.

[19] Goldstein RZ, Volkow ND. Drug addiction and its underlying neurobiologica basis: neuroimaging evidence for the involvement of the frontal cortex. Am J Psychiatry 2002;159:1642.

[20] Lubman DI, Yücel M, Pantelis C. Addiction, a condition of compulsive behaviour? Neuroimaging and neuropsychological evidence of inhibitory dysregulation. Addiction 2004;99:1491-502

[21] O'Brien CP, Childress AR, McLellan AT, Ehrman R. Classical conditioning in drug dependent humans. Ann N Y Acad Sci 1992;654:400-15.

[22] O’Brien CP, Childress AR, Ehrman R, Robbins SJ. Conditioning factors in drug abuse: can they explain compulsion. J Psychopharmacol 1998;12:15-22.

[23] De Wit H, Stewart J. Reinstatement of cocaine-reinforced responding in the rat. Psychopharmacology 1981;75:134-43.

[24] Shaham Y, Shalev U, Lu L, de Wit H, Stewart J. The reinstatement model of drug relapse: history, methodology and major findings. Psychopharmacology 2003;168:3-20.

[25] O’Brien CP, Childress C, McLellan AT, Ehrman R. A learning model of addiction. Res Publ Assoc Res Nerv Ment Dis 1992;70:157-77; Raven O'Brien CP, Childress C, McLellan AT, Ehrman R, Ternes JW. Types of conditioning found in drug-dependent humans. NIDA Res Monogr 1988;84:44-61.

[26] Everitt BJ, Robbins TW. Neural systems of reinforcement for drug addiction: from actions to habits to compulsion. Nat Neurosci 2005;8:1481-9.

[27] Boileau I, Dagher A, Leyton M, Gunn RN, Baker GB, Diksic M, et al. Modeling sensitization to stimulants in humans: an [11C] raclopride/positron emission tomography study in healthy men. Arch Gen Psychiatry 2006;63:1386-95.

[28] Vezina P, Leyton M. Conditioned cues and the expression of stimulant sensitization in animals and humans. Neuropharmacology 2009;56:160-8.

[29] DeWit H, Stewart J. Reinstatement of cocaine-reinforced responding in the rat. Psychopharmacology 1981:75:134-43.

[30] Field M, Mogg K, Bradley BP. Craving and cognitive biases for alcohol cues in social drinkers. Alcohol Alcohol 2005;40:504-10.

[31] Garland EL. Trait mindfulness predicts attentional and autonomic regulation of alcohol cue-reactivity. J Psychophysiol 2011;25:180-9.

[32] Marissen MA, Franken IH, Waters AJ, Blanken P, Van Den Brink W, Hendriks VM. Attentional bias predicts heroin relapse following treatment. Addiction 2006;101:1306-12.

[33] Waters AJ, Shiffman S, Sayette MA, Paty JA, Gwaltney CJ, Balabanis MH. Attentional bias predicts outcome in smoking cessation. Health Psychol 2003;22:378. 
[34] Carpenter KM, Schreiber E, Church S, McDowell D. Drug Stroop performance: relationships with primary substance of use and treatment outcome in a drug dependent outpatient sample. Addict Behave 2006;31:174-81.

[35] Waters AJ, Shiffman S, Bradley BP, Mogg K. Attentional shifts to smoking cues in smokers. Addiction 2003;98:1409-17.

[36] Kennedy AP, Gross RE, Ely T, Drexler KP, Kilts CD. Clinical correlates of attentional bias to drug cues associated with cocaine dependence. Am J Addict 2014;23:478-84

[37] Wilkinson D, Halligan P. The relevance of behavioural measures for functionalimaging studies of cognition. Nat Rev Neurosci 2004;5:67-73.

[38] Marhe R, Van de Wetering BJM, Franken IHA. Error-related brain activity predicts cocaine use after treatment at 3-month follow-up. Biol Psychiatry 2013;73:782-8.

[39] Maurage P, Pesenti M, Philippot P, Joassin F, Campanella S. Latent deleterious effects of binge drinking over a short period of time revealed only by electrophysiological measures. J Psychiatry Neurosci 2009;34:111-8.

[40] Field M, Munafo MR, Franken IH. A meta-analytic investigation of the relationship between attentional bias and subjective craving in substance abuse. Psychol Bull 2009;135:589-607.

[41] Fehr T, Wiedenmann P, Herrmann MD. Differences in ERP topographies during color matching of smoking-related and neutral pictures in smokers and nonsmokers. Int J Psychophysiol 2007;65:284-93.

[42] Herrmann MJ, Weijers HG, Wiesbeck GA, Aranda D, Böning J, Fallgatter AJ Event-related potentials and cue-reactivity in alcoholism. Alc Clin Exp Res 2000;24:1724-9.

[43] Namkoong K, Lee E, Lee CH, Lee BO, An SK. Increased P3 amplitudes induced by alcohol-related pictures in patients with alcohol dependence. Alc Clin Exp Res 2004;28:1317-23.

[44] Polich J, Kok A. Cognitive and biological determinants of P300: an integrative review. Biol Psychol 1995;41:103-46.

[45] Pontifex MB, Hillman CH, Polich J. Age, physical fitness, and attention: P3a and P3b. Psychophysiology 2009;46:379-87.

[46] Donchin E. Presidential address, 1980. Surprise!. Surprise? Psychophysiology 1981;18:493-513.

[47] Hajcak G, MacNamara A, Olvet DM. Event-related potentials, emotion, and emotion regulation: an integrative review. Dev Neuropsychol 2010;35: 129-55.

[48] Cuthbert BN, Schupp HT, Bradley MM, Birbaumer N, Lang PJ. Brain potentials in affective picture processing: covariation with autonomic arousal and affective report. Biol Psychol 2000;52:95-111.

[49] Lang PJ, Bradley MM, Cuthbert BN. Motivated attention: affect, activation, and action. Attention Orient Sensory Motiv Process 1997:97-135.

[50] Littel M, Euser AS, Munafò MR, Franken IHA. Electrophysiological indices of biased cognitive processing of substance-related cues: a meta-analysis. Neurosci Biobehav Rev 2012;36:1803-16.

[51] Nijs IMT, Franken IHA, Muris P. Food cue-elicited brain potentials in obese and healthy-weight individuals. Eat Behav 2008;9:462-70

[52] Lubman DI, Allen NB, Peters LA, Deakin JF. Electrophysiological evidence of the motivational salience of drug cues in opiate addiction. Psychol Med 2007:37:1203-9.

[53] American Psychiatric Association (APA). Diagnostic and statistical manual of mental disorders, DSM-IV. 4th ed. Washington (DC): American Psychiatric Association: 1994.

[54] National Institute on Alcohol Abuse and Alcoholism. Substance abuse and mental health services administration. Results from the 2004 National Survey on Drug Use and Health: National Findings. NIAAA; 2004. Available online at: http://www.oas.samhsa.gov/NSDUH/2k4NSDUH/2k4results/2k4results.htm\# fig7.3 [accessed March 2006].

[55] van den Wildenberg E, Wiers RW, Dessers J, Janssen RG, Lambrichs EH, Smeets $\mathrm{H}$, et al. A functional polymorphism of the $\alpha$-opioid receptor gene (OPRM1) influences cue-induced craving for alcohol in male heavy drinkers. Alc Clin Exp Res 2007;31:1-10.

[56] Saunders JB, Aasland OG, Babor TF, de la Fuente JR, Grant M. Development of the Alcohol Use Disorders Identification Test (AUDIT): WHO collaborative project on early detection of persons with harmful alcohol consumption-II. Addiction 1988;193:791-804.

[57] Spielberger CD, Gorsuch RL, Lusthene RE. Manual for the State and Trait Anxiety Inventory. Palo Alto: Consulting Psychologist Press; 1983.

[58] Beck AT, Steer RA, Brown GK. Manuel de l'inventaire de depression de Beck. 2eme edition Toronto, Ontario, Canada: The Psychological Corporation; 1998.

[59] Wiers RW, Eberl C, Rinck M, Becker ES, Lindenmeyer J. Retraining automatic action tendencies changes alcoholic patients' approach bias for alcohol and improves treatment outcome. Psychol Sci 2011;22:490-7.

[60] Spruyt A, De Houwer J, Tibboel H, Verschuere B, Crombez G, Verbanck P, et al. On the predictive validity of automatically activated approach/avoidance tendencies in abstaining alcohol-dependent patients. Drug Alcohol Depend 2013;127:81-6.

[61] Jasper H. Report of committee on methods of clinical exam in EEG. Electroencephalogr Clin Neurophysiol 1958;10:370-5

[62] Bertrand O, Perrin F, Pernier J. A theoretical justification of the average reference in topographic evoked potential studies. Electroencephalogr Clin Neurophysio 1985;62:462-4

[63] Polich J. Clinical application of the P300 event-related brain potential. Phys Med Rehabil Clin N Am 2004;15:133-61.

[64] Campanella S, Bruyer R, Froidbise S, Rossignol M, Joassin F, Kornreich C, et al. Is two better than one? A cross-modal oddball paradigm reveals greater sensitivity of the P300 to emotional face-voice associations. Clin Neurophysio 2010;12:1855-62

[65] Kareken DA, Claus ED, Sabri M, Dzemidzic M, Kosobud AEK, Radnovich A], et al. Alcohol-related olfactory cues activate the nucleus accumbens and ventral tegmental area in high-risk drinkers: preliminary findings. Alc Clin Exp Res 2004;28:550-7.

[66] Tapert SF, Brown GG, Baratta MV, Brown SA. fMRI BOLD response to alcohol stimuli in alcohol dependent young women. Addict Behav 2004;29: $33-50$.

[67] Mann K, Kiefer F, Smolka M, Gann H, Wellek S, Heinz A. Searching for responders to acamprosate and naltrexone in alcoholism treatment: rationale and design of the predict study. Alcohol Clin Exp Res 2009;33:674-83.

[68] Campanella S, Petit G, Verbanck P, Kornreich C, Noel X. How cognitive assessment through clinical neurophysiology may help optimize chronic alcoholism treatment. Clin Neurophysiol 2011;41:115-23.

[69] Domino EF. Effects of tobacco smoking on electroencephalographic, auditory evoked and event related potentials. Brain Cogn 2003;53:66-74

[70] Lehmann D, Strik WK, Henggeler B, Koenig T, Koukkou M. Brain electric microstates and momentary conscious mind states as building blocks of spontaneous thinking: I. Visual imagery and abstract thoughts. Int J Psychophysiol 1998;29:1-11

[71] Brunet D, Murray MM, Michel CM. Spatiotemporal analysis of multichannel EEG: CARTOOL. Comput Intell Neurosci 2011:813-70

[72] Tibshirani R, Walther G. Cluster validation by prediction strength. J Comput Graph Stat 2005;14:511-28.

[73] Pascual-Marqui RD, Esslen M, Kochi K, Lehmann D. Functional imaging with low resolution brain electromagnetic tomography (LORETA): review, new comparisons, and new validation. Jpn J Clin Neurophysiol 2002;30:81-94.

[74] Kim D-W, Shim M, Kim J-I, Im C-H, Lee S-H. Source activation of P300 correlates with negative symptom severity in patients with schizophrenia. Brain Topogr 2014;27:307-17.

[75] Collins DL, Neelin P, Peters TM, Evans AE. Automatic 3D intersubject registration of MR columetric data in standardized Talairach space. J Comput Assist Tomogr 1994;18:192-205.

[76] Evans AC, Collins DL, Millst SR, Brown ED, Kelly RL, Peters TM. 3D Statistica neuroanatomical models from 305 MRI volumes. IEEE Nucl Symp Med Image Conf 1993;95:1813-7.

[77] Lancaster JL, Woldorff MG, Parsons LM, Liotti M, Freitas CS, Rainey L, et al. Automated Talairach atlas labels for functional brain mapping. Hum Brain Mapp 2000;10:120-31.

[78] Littel M, Field M, van de Wetering BJM, Franken IHA. Reduced cognitive processing of alcohol cues in alcohol-dependent patients seeking treatment: an ERP study. J Exp Psychopathol 2013:4:291-302.

[79] Littel M, Franken IHA. The effects of prolonged abstinence on the processing of smoking cues: an ERP study among smokers, ex-smokers and never-smokers. J Psychopharmacol 2007:21(8):873-82.

[80] Robinson JD, Versace F, Engelmann JM, Cui Y, Slapin A, Oum R, et al. The motivational salience of cigarette-related stimuli among former, never, and current smokers. Exp Clin Psychopharmacol 2014, in press.

[81] Bauer LO. Electroencephalographic and autonomic predictors of relapse in alcohol dependent patients. Alcohol Clin Exp Res 1994;18:755-60.

[82] Winterer G, Klöppel B, Heinz A, Ziller M, Dufeu P, Schmidt LG, et al. Quantitative EEG (QEEG) predicts relapse in patients with chronic alcoholism and points to a frontally pronounced cerebral disturbance. Psychiatry Res 1998;78(1-2):101-13.

[83] Bauer LO. Predicting relapse to alcohol and drug abuse via quantitative electroencephalography. Neuropsychopharmacology 2001;25:332-40.

[84] Saletu-Zyhlarz GM, Arnold O, Anderer P, Oberndorfer S, Walter H, Lesch $\mathrm{OM}$, et al. Differences in brain function between relapsing and abstaining alcohol-dependent patients, evaluated by Eeg mapping. Alcohol Alcoho 2004;39:233-40.

[85] Marhe R, Luijten M, Franken IHA. The clinical relevance of neurocognitive measures in addiction. Front Psychiatry 2013;4:185

[86] Kober H, Mende-Siedlecki P, Kross EF, Weber J, Mischel W, Hart CL, et al. Prefrontal-striatal pathway underlies cognitive regulation of craving. Proc Nat Acad Sci 2010;107:14811-6.

[87] Littel M, Franken IHA. Implicit and explicit selective attention to smoking cues in smokers indexed by brain potentials. J Psychopharmacol 2011;25:503-13.

[88] Anton RF. Naltrexone for the management of alcohol dependence. N Engl J Med 2008;359:715-21.

[89] Beck A, Wüstenberg T, Genauck A, Wrase J, Schlagenhauf F, Smolka MN et al. Effect of brain structure, brain function, and brain connectivity on relapse in alcohol-dependent patients. Arch Gen Psychiatry 2012;6: 842-52.

[90] Fusar-Poli P, Placentino A, Carletti F, Landi P, Allen P, Surguladze S, et al. Functional atlas of emotional faces processing: a voxel-based meta-analysis of 105 functional magnetic resonance imaging studies. J Psychiatry Neurosci 2009:34:418-32

[91] Fujimaki N1, Miyauchi S, Pütz B, Sasaki Y, Takino R, Sakai K. Functional magnetic resonance imaging of neural activity related to orthographic, phonological, and lexico-semantic judgments of visually presented characters and words. Hum Brain Mapp 1999;8(1):44-59.

[92] Babiloni C, Del Percio C, Triggiani AI, Marzano N, Valenzano A, Petito A, et al. Attention cortical responses to enlarged faces are reduced in underweight subjects: an electroencephalographic study. Clin Neurophysiol 2011;122:1348-59. 
[93] Clark VP, Fannon S, Lai S, Benson R, Bauer L. Responses to rare visual target and distractor stimuli using event-related fMRI. J Neurophysiol 2000;83:3133-9.

[94] Kiehl KA, Laurens KR, Duty TL, Forster BB, Liddle PF. An event-related fMRI study of visual and auditory oddball tasks. J Psychophysiol 2001;15:221-40.

[95] Proverbio AM, Adorni R, Zani A, Trestianu L. Sex differences in the brain response to affective scenes with or without humans. Neuropsychologia 2009;47:2374-88.

[96] Lee J-H, Lim Y, Wiederhold BK, Graham SJ. A functional magnetic resonance imaging (fMRI) study of cue-induced smoking craving in virtual environments. Appl Psychophys Biol 2005;30:195-204.
[97] Beck A, Wüstenberg T, Genauck A, Wrase J, Schlagenhauf F, Smolka MN, et al. Effect of brain structure, brain function, and brain connectivity on relapse in alcohol-dependent patients. Arch Gen Psychiatry 2012;69:842-52.

[98] Campanella S, Bourguignon M, Peigneux P, Metens T, Nouali M, Goldman $\mathrm{S}$, et al. BOLD response to deviant face detection informed by P300 eventrelated potential parameters: a simultaneous ERP-fMRI study. NeuroImage 2013:71:92-8.

[99] Johnson BA, Cloninger CR, Roache JD, Bordnick PS, Ruiz P. Age of onset as a discriminator between alcoholic subtypes in a treatment-seeking outpatient population. Am J Addict 2000;9:17-27. 